\title{
HOW TO APPLY ICA ON ACTUAL DATA ? EXAMPLE OF MARS HYPERSPECTRAL IMAGE ANALYSIS.
}

\author{
Christian Jutten ${ }^{1}$, Saïd Moussaoui ${ }^{2}$, Frédéric Schmidt ${ }^{3}$ \\ (1) GIPSA-lab, Dept. Images and Signal, Grenoble, France \\ (2) Institut de Recherche en Communication et Cybernétique de Nantes, Nantes, France \\ (3) Laboratoire de Planétologie de Grenoble, Saint-Martin d'Hères, France
}

\begin{abstract}
As any estimation method, results provided by ICA are dependent of a model - usually a linear mixture and separation model - and of a criterion - usually independence. In many actual problems, the model is a coarse approximation of the system physics and independence can be more or less satisfied, and consequently results are not reliable. Moreover, with many actual data, there is a lack of reliable knowledge on the sources to be extracted, and the interpretation of the independent components (IC) must be done very carefully, using partial prior information and with interactive discussions with experts. In this talk, we explain how such a scientific method can take place on the example of analysis of Mars hyperspectral images.
\end{abstract}

Index Terms - source separation, independent component analysis, hyperspectral images, Mars Express, positivity, Bayesian source separation

\section{INTRODUCTION}

Currently, independent component analysis (ICA) is a very popular method for solving blind source separation problems. Its success is probably due to a good theoretical framework and especially to a very large range of applications (see in $[1,2])$, in many domains like biomedical signal processing, audio signal processing, communications, smart sensor arrays, hyperspectral image processing, etc.

So, an important question is the following : can one apply ICA like a simple blackbox ? In other works, can one be confident of the results provided by ICA ?

In fact, such a question is very usual for any estimation method. And ICA is nothing but a particular one. Basically, any estimation method is based on three ingredients: a parametric model, a criterion and an optimization algorithm. The parametric model provides a simple representation and

Correspondance concerning this paper must be sent to Christian Jutten, GIPSA-lab, 46 avenue Félix Viallet, 38031 Grenoble Cedex, Christian.Jutten@inpg.fr restricts the solution to a particular space. The criterion, statistical indedependence for ICA methods, is a measure of the quality of the solution. The optimization algorithm is the way for optimizing (usually minimizing or maximizing) the criterion in the parametric model space. If we assume the optimization algorithm does not stop in spurious local extrema, ICA algorithms always converges to a solution which will be optimal with respect to the chosen criterion in the parametric model space.

When applying ICA, we consider $n$-dimensional observation vector, $\boldsymbol{x}(t)$, which are assumed to be mixtures - through an unknown function $\mathcal{F}$ from $\mathbb{R}^{n}$ to $\mathbb{R}^{p}$ - of $p$-dimensional unobserved sources, $s(t)$, assumed to be statistically independent:

$$
\boldsymbol{x}(t)=\mathcal{F} \boldsymbol{s}(t) .
$$

In the following, we assume there are more observations than sources $(n \leq p)$ and $\mathcal{F}$ is invertible. Then, ICA methods result in estimating a separating transform $\mathcal{G}$ which provides:

$$
\boldsymbol{y}(t)=\mathcal{G}(\boldsymbol{x}(t))=(\mathcal{G} \circ \mathcal{F})(s(t)),
$$

with statistically independent components. Theoretical results prove that independence is sufficient for estimating the unknown sources, especially for linear mixtures, instantaneous [3] or convolutive $[4,5,6]$, and even for particular nonlinear mixtures $[7,8,9,10,11]$.

Of course, the estimated transform $\mathcal{B}$, and sources $\boldsymbol{y}(t)$, even optimal in the sense of the criterion, leads to relevant solution if:

- the nature of the separating transform $\mathcal{G}$ is suited to the mixing model $\mathcal{F}$,

- the independence assumption is actually satisfied by the unknown sources, $s(t)$.

On the contrary, i.e. when the mixing model is wrong (and then the separating model) or the independence assumption is wrong, ICA can lead to irrelevant results. 
Practically, in actual applications, for applying successfully ICA, one has first to model, according to the physics of the system, the relationships between the observations and the sources, and to check if the independence assumption of sources is realistic. Of course, the model as well as the independence assumption are usually approximations. If approximations is too coarse, ICA can lead to irrelevant results.

In this paper, we illustrate these general ideas on an actual application in Astrophysics: the analysis of Mars hyperspectral images, whose the goal is to classify the planet Mars surface (see [12] for details). The paper is organized as follows. In Section 2, we derive a mixing model of observations (hyperspectral images) based on physics. In Section 3, we consider two possible ICA models. Section 4 presents the ICA results and discuss them. Section 5 show how one can improve results, taking into account extra informations, here positivity. Conclusions are summarized in Section 6.

\section{PHYSICAL MODEL OF HYPERPSECTRAL IMAGES}

The OMEGA spectrometer, carried by Mars Express spacecraft on an elliptical orbit, has a spatial resolution range from $300 \mathrm{~m}$ to $4 \mathrm{~km}$. This instrument has three channels, a visible channel and two near infrared (IR) channels. In this paper, we focus on a data set consisting of a single hyperspectral data cube of the South Polar Cap of Mars in the local summer where $\mathrm{CO}_{2}$ ice, water ice and dust were previously detected [13, 14]. This data cube consists of 2 IR channels: 128 spectral planes from $0.93 \mu \mathrm{m}$ to $2.73 \mu \mathrm{m}$ with a resolution of $0.013 \mu \mathrm{m}$ and 128 spectral planes from $2.55 \mu \mathrm{m}$ to $5.11 \mu \mathrm{m}$ with a resolution of $0.020 \mu \mathrm{m}$. After calibration, the dimensionless physical unit used to express the spectra is the "reflectance", which is the ratio between the irradiance leaving each pixel toward the sensor and the solar irradiance at the ground. Interactions between photons coming from the sun and the planet Mars, through its atmosphere and surface, allows us to identify the different compounds present in the planet. Those compounds are mixed and usually different chemical species can be identified in each measured spectra. Two kinds of physical mixing at the ground can be observed [15]:

- Geographic mixture: each pixel is a patchy area made of several pure compounds. This type of mixture happens when the spatial resolution is not large enough to observe the complex geological combination pattern. The total reflectance in this case will be a weighted sum of the pure constituent reflectances. The weights (abundance fractions) associated to each pure constituent are surface proportions inside the pixel.

- Intimate mixture: each pixel is made of one single terrain type which is a mixture at less than the typical mean-path scale (typically the order of $1 \mathrm{~mm}$ scale). The total reflectance in this case will be a nonlinear function of pure constituent reflectances.

In this paper, we restrict our analysis with hypothesis of a geographical mixtures and hence linear mixing models, i.e. $\mathcal{F}$ reduces to a matrix.

\subsection{Observation Model}

Under geographic mixture model and acceptable assumptions concerning atmospheric contributions [16], the radiance factor at location $(x, y)$ and at wavelenght $\lambda$ is:

$$
\begin{aligned}
& L(x, y, \lambda)= \\
& \quad\left(\rho_{a}(\lambda)+\Phi(\lambda) \sum_{p=1}^{P} \alpha_{p}(x, y) \rho_{p}(\lambda)\right) \cos [\theta(x, y)]
\end{aligned}
$$

where $\Phi(\lambda)$ is the spectral atmospheric transmission, $\theta(x, y)$ the angle between the solar direction and the surface normal (solar incidence angle), $P$ the number of endmembers in the region of coordinates $(x, y), \rho_{p}(\lambda)$ the spectrum of the $p$ th endmember, $\alpha_{p}(x, y)$ its weight in the mixture and $\rho_{a}(\lambda)$ the radiation that does not arrive directly from the area under view. This mixture model can also be written as:

$$
L(x, y, \lambda)=\sum_{p=1}^{P} \alpha_{p}^{\prime}(x, y) \cdot \rho_{p}^{\prime}(\lambda)+E(x, y, \lambda)
$$

where

$$
\left\{\begin{array}{l}
\alpha_{p}^{\prime}(x, y)=\alpha_{p}(x, y) \cos [\theta(x, y)] \\
\rho_{p}^{\prime}(\lambda)=\Phi(\lambda) \rho_{p}(\lambda) \\
E(x, y, \lambda)=\rho_{a}(\lambda) \cos [\theta(x, y)]
\end{array}\right.
$$

As it can be seen in equation (5), the true endmember spectra are affected by the atmospheric attenuation and the abundance fractions are corrupted by the solar angle effect. Clearly, the spectra obtained are ideally the spectra of the endmembers with atmospheric contribution. On the contrary, it is attempted to correct the solar angle effect to give a map of the constituent proportions in the observed area. In fact, since the abundance fraction is proportional to the quantity of each constituent in the geographical mixture, it can be deduced from the mixture model (4) and equation (5) that the abundance fractions are not altered by the geometrical effect since:

$$
\begin{aligned}
c_{p}(x, y) & =\frac{\alpha_{p}^{\prime}(x, y)}{\sum_{j=1}^{P} \alpha_{j}^{\prime}(x, y)}, \\
& =\frac{\alpha_{p}(x, y)}{\sum_{j=1}^{P} \alpha_{j}(x, y)} .
\end{aligned}
$$




\subsection{Data Size}

Practically, the data sets consist of 174 channels collected in the infrared region: noisy, hot and dead spectels have been excluded. The spatial size of the data sets varies from $323 \leq$ $N_{x} \leq 751$ and $N_{y} \in\{64,128\}$, for a total pixel number: $41344 \leq N_{x} \cdot N_{y} \leq 56832$. Each data set (i.e. hyperspectral image) is then a data cube of size $N_{x} \times N_{y} \times N_{f}$ which contains between $7,193,856$ and $9,888,768$ according to the image size.

\section{DECOMPOSITION MODELS BASED ON ICA}

Let us now consider a hyperpectral data cube with $N_{f}$ images of $N_{z}=\left(N_{x} \times N_{y}\right)$ pixels obtained from $N_{f}$ frequency bands. For simplicity, assume raw vectorized images $I\left(n, \lambda_{k}\right)$, with $1 \leqslant n=(i-1) N_{y}+j \leqslant N_{z}$ (where $i$ and $j$ are the initial row and column image indices) is the spatial index and $k, k=1, \ldots, N_{f}$, is the spectral index for wavelength $\lambda_{k}$. Consequently, two representations of the hyperspectral data can be considered: spectral and spatial mixture models.

\subsection{Spectral Mixture Model:}

Each pixel of spatial index $n$ gives an observed spectrum of $N_{f}$ frequency samples, which is represented by the linear approximation:

$$
I_{n}\left(\lambda_{k}\right) \approx \sum_{p=1}^{N_{c}} a_{(n, p)} \psi_{p}\left(\lambda_{k}\right), \quad \forall n=1, \ldots, N_{z},
$$

where $\psi_{p}\left(\lambda_{k}\right)$, for $p=1, \ldots, N_{c}$, are the constituent reflectance spectra, and the number $N_{c}$ is chosen according to the desired accuracy of the approximation. Denoting the vectorized image (of dimension $\left.N_{x} \times N_{y}\right) \mathbf{I}\left(\lambda_{k}\right)$, the $\left(N_{z} \times N_{c}\right.$ ) mixing matrix $\boldsymbol{A}$ and $\boldsymbol{\Psi}\left(\lambda_{k}\right)=\left[\psi_{1}\left(\lambda_{k}\right), \ldots, \psi_{N_{c}}\left(\lambda_{k}\right)\right]^{T}$, this spectral mixture model is then expressed as:

$$
\mathbf{I}\left(\lambda_{k}\right) \approx \boldsymbol{A} \cdot \boldsymbol{\Psi}\left(\lambda_{k}\right) .
$$

Practically, this spectral model intends to approximate the spectrum of each pixel as a sum of $N_{c}$ component spectra of the area corresponding to this pixel coordinates. If ICA is used for the estimation, then the $N_{c}$ basis spectra $\psi_{p}, p=$ $1, \ldots, N_{c}$, should be statistically independent. Moreover, the $p$-th column of the matrix $\boldsymbol{A}$ is the unfolded image associated to the basis spectrum $\psi_{p}$. According to this model, we have $N_{x} \cdot N_{y} \approx 50,000$ sensors and a small number of samples $N_{f}=174$, for estimating the large matrix $\boldsymbol{A}$ which has $N_{z} \times N_{c} \approx 250,000$ parameters (taking $N_{c}=5$ ).

\subsection{Spatial Mixture Model:}

This model assumes that for each wavelength $\lambda_{k}$, the measured image $I_{\lambda_{k}}(n)$ is a weighted sum of $N_{c}$ basis images, denoted $I I_{p}(n), p=1, \ldots, N_{c}$ :

$$
I_{\lambda_{k}}(n) \approx \sum_{p=1}^{N_{c}} b_{\left(\lambda_{k}, p\right)} I I_{p}(n), \quad \forall k=1, \ldots, N_{f} .
$$

In vector notations, denoting the $N_{f} \times N_{c}$ matrix $\boldsymbol{B}$ and $\mathbf{I I}(n)=\left[I I_{1}(n), \ldots, I I_{N_{c}}(n)\right]^{T}$, one can write:

$$
\mathbf{I}(n) \approx \boldsymbol{B} \cdot \mathbf{I I}(n) .
$$

Practically, this spatial model intends to approximate the whole image at each frequency as a sum of $N_{c}$ basis images. If ICA is used for the estimation, then the $N_{c}$ basis images $I I_{p}$, $p=1, \ldots, N_{c}$, should be statistically independent. Moreover, the $k$-th column of the matrix $\boldsymbol{B}$ is the spectrum associated to the basis image $I I_{k}$. According to this model, we have $N_{f}=174$ sensors, and a very large number of samples $N_{z} \approx 50,000$ for estimating the matrix $\boldsymbol{B}$ which has $N_{f} \times N_{c}<900$ parameters (taking $N_{c}=5$ ).

Comment on the notations: For limiting notation complexity, in the two models, the hyperspectral dataset is always denoted I, but, in the spatial model, one considers the dataset like a $N_{f} \times N_{z}$ matrix while it is a $N_{z} \times N_{f}$ matrix in the spectral model. The two matrices contain exactly the same entries, but are in fact transposed from a model to the other one.

\subsection{Reference Data and Classification}

Since no ground truth is possible on Mars, we need some reference informations about the three main endmembers: dust, $\mathrm{CO}_{2}$ and $\mathrm{H}_{2} \mathrm{O}$ ices. Two kinds of reference data are available (Fig. 1): reference classification masks obtained from supervised classification study [14], and reference spectra. Reference spectra of $\mathrm{CO}_{2}$ ice and $\mathrm{H}_{2} \mathrm{O}$ ice are simulations produced by a radiative transfer model in typical physical conditions of the Permanent South Polar Cap of Mars [17]. These two reference spectra are atmosphere free simulations. The dust reference spectrum is derived from an OMEGA's observation. Consequently, this reference spectrum contains the atmospheric transmission. The wavanglet classification method produces classification masks (Fig. 1, (d) to (f)) which are neither unique nor complete, i.e. pixels can be in more than one class and not all the pixels are classified.

\section{ICA RESULTS ON HYPERSPECTRAL IMAGES}

In the framework of hyperspectral data, following the mixture model (4), one considers the data can be viewed as a linear mixtures of sources. ICA provides such a model where the sources are mutually independent. In this Section, we consider the spatial mixture model (9) and we use the well known ICA algorithm, JADE, based on the joint approximate diagonalization of cumulant matrices [18]. 


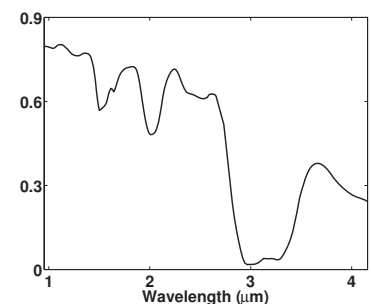

(a) $\mathrm{CO}_{2}$

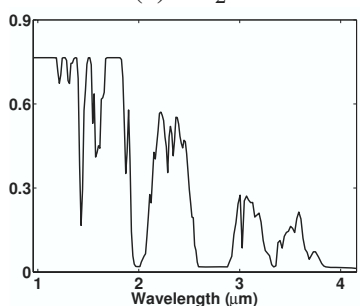

(b) $\mathrm{H}_{2} \mathrm{O}$

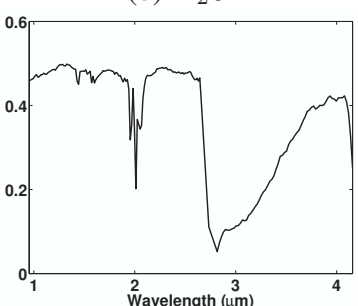

(c) Dust

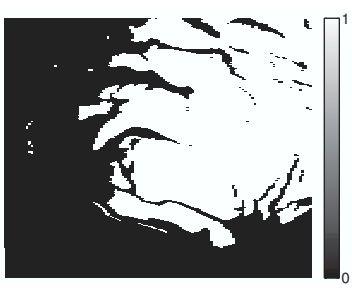

(d) $\mathrm{CO}_{2}$

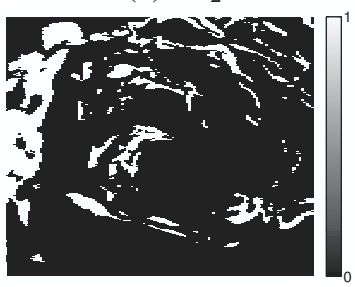

(e) $\mathrm{H}_{2} \mathrm{O}$

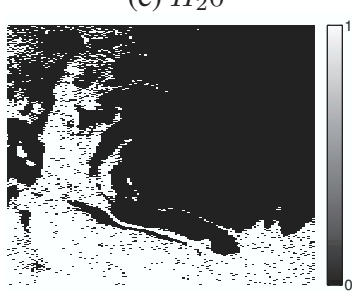

(f) Dust
Fig. 1. Reference spectra (left) and classification masks (right)

\subsection{Spatial ICA}

In this experiment, we use two sets of data. The first one is the original data set (RDS, for raw data set), while the second one is a preprocessed (done by astrophysicists) data set (PDS), obtained from the original data set by canceling the geometrical effect, atmospheric attenuation and a few known defects of the sensors.

Choosing the number of ICs: a first step is to chose the number, $N_{c}$, of independent component (IC), at least equal to the number of sources present in the mixtures. If $N_{c}$ is larger, the accuracy of the approximation (9) increases, but extra ICs can be difficult to interpret. This is done using principal component analysis: on RDS, with 7 principal components, $98.58 \%$ of the variance of the initial image is preserved. In figure 2, we show the 7 ICs estimated with JADE from the original data set.

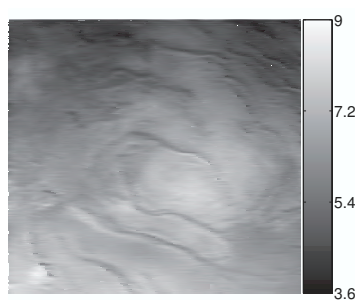

(a) IC1

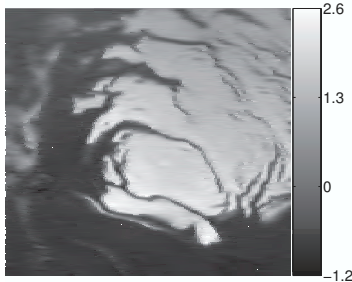

(b) IC2

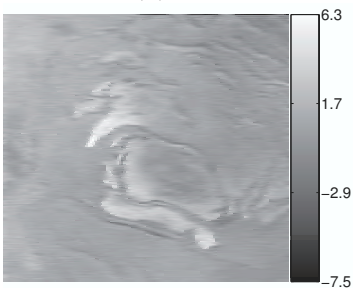

(d) IC4

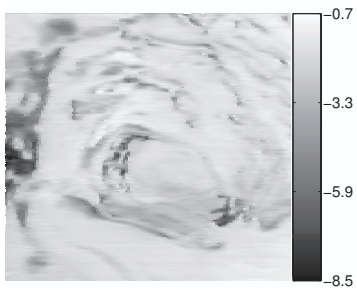

(f) IC6

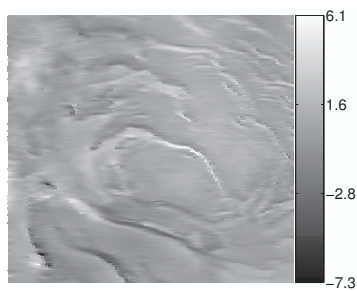

(c) IC3

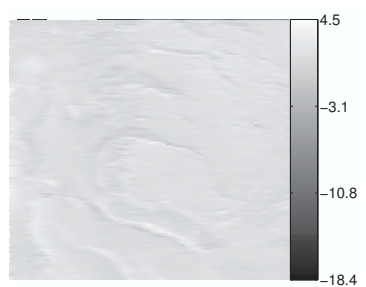

(e) IC5

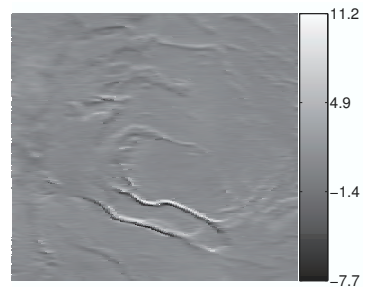

(g) IC7
Fig. 2. Independent components of the RDS hyperspectral image of south polar cap computed with JADE with 7 ICs.

Relevance of the ICs: a second step consists in evaluating the relevance of each component $I C_{k}$ in the approximation. This is done by measuring the relative quadratic loss:

$$
\epsilon_{k}=-10 \log _{10}\left(\frac{P_{\hat{I}_{N_{c}}}-P_{\hat{I}_{N_{c} \mid N_{k}}}}{P_{\hat{I}_{N_{c}}}}\right),
$$

obtained when replacing the $N_{c}$-order approximation $\hat{I}_{N_{c}}$ by with the $\left(N_{c}-1\right)$-order, denoted $\hat{I}_{N_{c} \mid N_{k}}$ obtained by canceling the $I C_{k}$, and where the energies of the approximated 
images are computed as:

$$
\begin{aligned}
P_{\hat{I}_{N_{c}}} & =\sum_{m=1}^{N_{f}} \sum_{n=1}^{N_{z}}\left(\sum_{p=1}^{N_{c}} b_{\left(\lambda_{m}, p\right)} I I_{p}(n)\right)^{2} \\
P_{\hat{I}_{N_{c} \mid N_{k}}} & =\sum_{m=1}^{N_{f}} \sum_{n=1}^{N_{z}}\left(\sum_{p=1, p \neq k}^{N_{c}} b_{\left(\lambda_{m}, p\right)} I I_{p}(n)\right)^{2}(13)
\end{aligned}
$$

These values, computed for the ICs estimated with the two sets of data, are given in table 1 .

Finally, one has to wonder if the ICs are relevant and especially if they are robust with respect to the ICA algorithm, to similar images and to the number of ICs. In this purpose, we did three sets of experiments:

1. we compared results obtained with the two sets of data (RDS and PDS). We checked that a high quality reconstruction is achieved with 4 ICs for PDS and with 7 ICs for RDS. If more than 4 (or 7) ICs are used, one always get the same main 4 (or 7) ICs, the others have very small contributions on the image reconstruction and cannot be interpreted. For this reason, we chose 4 ICs for PDS and 7 ICs with RDS ;

2. in both data sets, images are resized using line subsampling (one line over two is kept). Then, this provides two different but very similar images that we call odd image and even image. We can check that ICA (with different IC numbers) of matched odd and even image leads to almost rigorously equal results, which again shows the reliability of the extracted ICs ;

3. we run three algorithms: FastICA [1] with various nonlinearities in the symmetric or deflation versions and JADE [18]. In all the experiments, one obtained very close results (ICs and reconstruction performance) with JADE and symmetric FastICA, while performance of deflation FastICA was worse. JADE is preferred since it has a low computational load and requires any parameter, except a stopping criterion.

\subsection{ICs interpretation:}

In the spatial approximation, each $\mathrm{IC}_{k}$ can be viewed as an image, while the column $k$ of the mixing matrix is the spectrum related to $\mathrm{IC}_{k}$. So, IC interpretation can be done comparing the IC image or spectra to the reference classification masks or reference spectra (Fig. 1). Computing correlation with classification masks, the components $\mathrm{IC}_{2}$ and $\mathrm{IC}_{6}$ can be easily identified (table 1) to respectively $\mathrm{CO}_{2}$ ice and $\mathrm{H}_{2} \mathrm{O}$ ice. In addition, correlation with reference spectra seems to confirm (partly) this interpretation. Conversely, the spectrum associated to $\mathrm{IC}_{4}$ has typical bands of both dust, $\mathrm{CO}_{2}$ and $\mathrm{H}_{2} \mathrm{O}$ ices. We could interpret this IC as a nonlinear intimate mixture effect or a non independent distribution of those components.
The other four components $\left(\mathrm{IC}_{1}, \mathrm{IC}_{3}, \mathrm{IC}_{5}\right.$ and $\left.\mathrm{IC}_{7}\right)$ cannot be interpreted with spectral informations. Using the preprocessed data, we remark that the energies of these ICs are very small (Table 1, last column), i.e. these ICs, strongly reduced by the preprocessing, must be related to phenomena cancelled by the preprocessing. In fact, IC1 (Fig. 2(a)) has a luminance gradient which is characteristic of the solar angle effect which should be the $E(x, y, \lambda)$ term in equation (5). $\mathrm{IC}_{7}$ (Fig. 2(g)) looks like a high-pass filter mainly on the $y$ direction of the image. This along track direction maximizes the instrument shift between the two near IR detectors. This effect is independent of the spectra model and thus it is detected as a separate IC and can be used to assess the quality of the preprocessing. $\mathrm{IC}_{3}$ could be associated to the transmission in the atmosphere effect because it is similar to a map of topography. At first glance, $\mathrm{IC}_{5}$ (Fig. 2(e)) was not recognized. But at a closer look, the first line in the image has a very low response, and corresponds to a corrupted line in the dataset, due to a known sensor failure.

\subsection{Classification}

From $\mathrm{IC}_{2}$ and $\mathrm{IC}_{6}$ interpretation, we deduce classification masks of $\mathrm{CO}_{2}$ and $\mathrm{H}_{2} \mathrm{O}$ ices with an easy criteria : if the IC has a positive value then $\mathrm{CO}_{2}$ ice - respectively $\mathrm{H}_{2} \mathrm{O}$ ice - is detected. The classification results compared with the reference classification is seen in table 2. False alarm and missing indicate the differences between the classification based on the ICs with respect to that based on wavanglet classification. However, keep in mind that the reference images, although pertinent, are not the ground truth and thus the false classifications in this case are not necessarily false.

An interesting result is that the classification of the original dataset is slighly better than the classification of the pre-

\begin{tabular}{llccc}
\hline$k$ & Identification & Figure & $\begin{array}{c}\text { RDS data } \\
\epsilon_{k}[\mathrm{~dB}]\end{array}$ & $\begin{array}{c}\text { PDS data } \\
\epsilon_{k}[\mathrm{~dB}]\end{array}$ \\
\hline \hline 1 & Solar angle effect & $2(\mathrm{a})$ & 32.6 & 1.3 \\
2 & $\mathrm{CO}_{2}$ ice & $2(\mathrm{~b})$ & 16.3 & 10.7 \\
3 & Atmospheric effect & $2(\mathrm{c})$ & 12.2 & 0.88 \\
4 & Intimate mixture & $2(\mathrm{~d})$ & 6.8 & 6.6 \\
5 & Corrupted line & $2(\mathrm{e})$ & 6.2 & - \\
6 & $\mathrm{H}_{2} \mathrm{O}$ ice & $2(\mathrm{f})$ & 7.1 & 5.9 \\
7 & Channel shift & $2(\mathrm{~g})$ & 2.0 & 0.1 \\
\hline
\end{tabular}

Table 1. Independent Components estimated with JADE. First column indicates the number of the IC. IC interpretation (see text for details) is given in column two. The third column refers to the Fig. number (from 2(a) to $2(\mathrm{~g})$ ). The fourth and fifth column are the loss in $d B\left(\epsilon_{k}\right)$ obtained if $\mathrm{IC}_{k}$ is not used in the approximation, for raw or preprocessed data, respectively. 


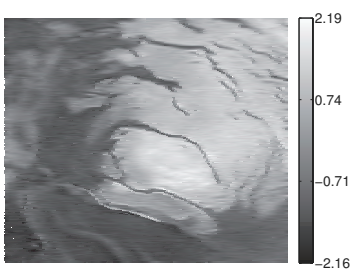

(a) IC related to $\mathrm{CO}_{2}$ ice

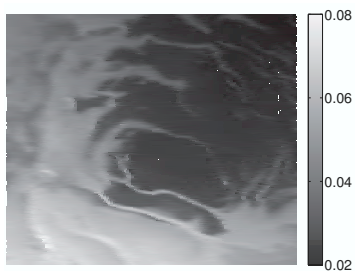

(c) $1.9833 \mu \mathrm{m}$

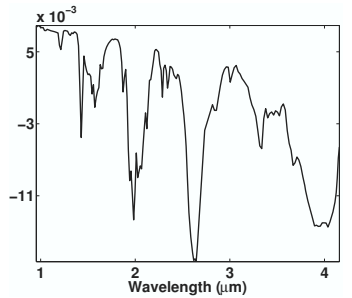

(b) IC2 mixing coefficients

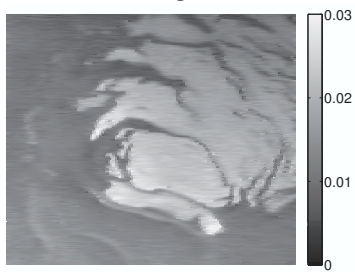

(d) $2.9833 \mu \mathrm{m}$
Fig. 3. IC2 is a mixture of $\mathrm{CO}_{2}$ ice and dust

processed dataset. This is in accordance to the SNR gained in table 1 , since the atmospheric removal seems to take energy in the $\mathrm{CO}_{2}$ and $\mathrm{H}_{2} \mathrm{O}$ ice components.

\subsection{Dependence of Spatial IC}

Clearly, looking at the reference spectra and reference images, it appears that the fundamental assumption of independence is not at all satisfied neither in spectral nor in spatial dimensions. The spatial independence can be tested by looking at the covariance between the reference classification masks (Fig. 1).

$R_{s}\left\{I_{\text {dust }}, I_{\mathrm{CO}_{2}}, I_{\mathrm{H}_{2} \mathrm{O}}\right\}=\left(\begin{array}{ccc}1 & -0.61 & -0.24 \\ -0.61 & 1 & -0.25 \\ -0.24 & -0.25 & 1\end{array}\right)$

When using spatial ICA, components of $\mathrm{CO}_{2}$ and $\mathrm{H}_{2} \mathrm{O}$ ices are retrieved but dust does not appear as a separate component as seen on Fig. 2. The negative correlation seen in (14) is not surprising when looking at Fig. 1 (d, e and f). Spatially, dust and $\mathrm{CO}_{2}$ ice are strongly complementary, and

\begin{tabular}{lccc}
\hline & Correct & Missing & False alarm \\
\hline \hline \multicolumn{2}{l}{ Original Data } \\
$\mathrm{CO}_{2}$ & $97.1 \%$ & $1.1 \%$ & $1.8 \%$ \\
$\mathrm{H}_{2} \mathrm{O}$ & $91.3 \%$ & $8.7 \%$ & $1.0 \%$ \\
\hline \hline \multicolumn{2}{l}{ Preprocessed Data } \\
$\mathrm{CO}_{2} 95.9 \%$ & $2.0 \%$ & $2.1 \%$ \\
$\mathrm{H}_{2} \mathrm{O}$ & $90.0 \%$ & $8.1 \%$ & $1.9 \%$ \\
\hline
\end{tabular}

Table 2. Classification of $\mathrm{CO}_{2}$ and $\mathrm{H}_{2} \mathrm{O}$ ices compared with the reference classification even more than the reference masks indicate. As a result, dust is not retrieved when using spatial ICA as a separate IC, but is frequently recovered as the negative of $\mathrm{CO}_{2}$ ice.

Coming back to IC2, which corresponds to $\mathrm{CO}_{2}$ ice (Fig. 4(a)), and look at its related spectrum (i.e. the column 2 of $\boldsymbol{B}$ ) on Fig. 3). In fact, as clearly shown in Fig. 3(b), this column, although correlated to $\mathrm{CO}_{2}$ spectrum, takes both positive and negative values: when the mixing coefficients take a positive value, for example at $2.98 \mu \mathrm{m}$, the dominating element for this wavelength is $\mathrm{CO}_{2}$ ice as seen on Fig. 3(d); on the contrary, when the mixing coefficients take a negative value as at $1.98 \mu \mathrm{m}$ the dominating element is dust as seen of figure 3(d). Finally, since the column 2 of $\boldsymbol{B}$ takes both positive and negative values, it cannot be interpreted as a spectrum.

\subsection{Discussion}

At first glance, spatial ICA provides ICs which can be interpreted as artifacts or endmembers. Concerning artifact ICs, the results are very interesting, and suggest that the data preprocessing could be avoided and done using ICA results. In fact, the classification performance seems ${ }^{1}$ even a little bit better on original data than on preprocessed data. Conversely, only two endmembers $\left(\mathrm{CO}_{2}\right.$ and $\mathrm{H}_{2} \mathrm{O}$ ices $)$ are associated to $\mathrm{IC}_{2}$ and $\mathrm{IC}_{6}$ : the third one, dust, mainly appears as the negative on $\mathrm{CO}_{2}$, which results in a very poor classification of dust. Moreover, the decomposition done by spatial ICA leads to a matrix $\boldsymbol{B}$ whose columns are not positive, and consequently cannot be considered as spectra. Finally, the IC statistical independence, the hypothesis on which ICA is based, is not satisfied. Especially, in the endmember classification, it appears that dust and $\mathrm{CO}_{2}$ ice are strongly correlated. Thus, the reliability of ICA is not sure, and the relevance of the extracted ICs is poor. Consequently, other methods, based on priors satisfied by the data, must be investigated.

\section{BEYOND ICA}

The main constraint in data decomposition of hyperspectral mixture is the positivity of both the mixing coefficients and the source signals. Unfortunately, this constraint alone does not lead to a unique solution unless under some particular conditions $[19,20]$. Thus, in general cases, additional assumptions are required to select a particular solution among the admissible ones. The estimation can then be performed using either a constrained least square $[21,22,23,24]$ or a penalized least square $[25,26,27]$ estimation. Unlike the constrained least squares methods, the penalized approaches lead to an unconstrained optimization problem and ensures the uniqueness of the solution for a fixed set of the regularization parameters. However, one can address the problem with regularization in the more general Bayesian framework.

\footnotetext{
${ }^{1}$ there is no ground truth, but only a reference classification: "better performance" only means "better correlation" with the reference classification.
} 


\subsection{Bayesian Positive Source Separation}

In fact, in a Bayesian source separation approach, one can ideally incorporate any prior knowledge as long as the prior can be stated in probabilistic terms. The approach is founded on the likelihood $p(\boldsymbol{x} \mid \boldsymbol{A}, \boldsymbol{s})$ and prior distributions of the source signals and mixing coefficients. Applying Bayes' theorem leads to:

$$
p(\boldsymbol{A}, \boldsymbol{s} \mid \boldsymbol{x}) \propto p(\boldsymbol{x} \mid \boldsymbol{A}, \boldsymbol{s}) \times p(\boldsymbol{A}) \times p(\boldsymbol{s}) .
$$

From this posterior law, both the mixing matrix $\boldsymbol{A}$ and sources $s$ can be estimated using various Bayesian estimators. A complete discussion on Bayesian approach to source separation can be found in $[28,29,30]$. However, its application to the case of positive sources and mixing has only received a few attention $[31,32,33]$. In this purpose, a recent contribution consists of the method termed by Bayesian positive source separation (BPSS) [34, 35], which allows to jointly estimate source signals, mixing coefficients and regularization parameters in an unsupervised framework.

\subsection{Separation with positivity constraint}

Here, due to strong spatial correlation between endmembers $\left(\mathrm{CO}_{2}\right.$ and dust), we propose to consider a spectral decomposition:

$$
I_{n}\left(\lambda_{k}\right)=\sum_{p=1}^{N_{c}} a_{(p, n)} \psi_{p}\left(\lambda_{k}\right)+E_{n}\left(\lambda_{k}\right) \quad \forall n=1, \ldots, N_{z},
$$

where $E_{n}\left(\lambda_{k}\right)$ is a noise term which models errors due to the simplified model (4), the restricted number of components, $N_{c}$ and measurement noise, the Bayesian model is then summarized as:

$$
\begin{aligned}
\left(E_{n}\left(\lambda_{k}\right) \mid \sigma_{n}^{2}\right) & \sim \mathcal{N}\left(E_{n}\left(\lambda_{k}\right) ; 0, \sigma_{n}^{2}\right), \\
\left(\psi_{p}\left(\lambda_{k}\right) \mid \alpha_{p}, \beta_{p}\right) & \sim \mathcal{G}\left(\psi_{p}\left(\lambda_{k}\right) ; \alpha_{p}, \beta_{p}\right), \\
\left(a_{(p, n)} \mid \gamma_{p}, \delta_{p}\right) & \sim \mathcal{G}\left(a_{(p, n)} ; \gamma_{p}, \delta_{p}\right),
\end{aligned}
$$

where $\mathcal{N}\left(z ; 0, \sigma^{2}\right)$ denotes a Gaussian distribution of the random variable $z$ with zero mean and variance $\sigma^{2}$ and $\mathcal{G}(z ; \alpha, \beta)$ stands for a Gamma distribution of the random variable $z$ with parameters $(\alpha, \beta)$. The Gamma law takes into account explicitly the positivity constraint since the probability distribution is zero for negative values: (17) and (18) insure then the positivity of spectra and mixing matrix entries. In addition, its two parameters give a flexibility to adapt its shape to that of spectral source signals. According to this probabilistic model and Bayes' theorem, with the hypothesis of statistical independence of the source signals and the mixing coefficients, the joint a posteriori distribution becomes:

$$
\begin{aligned}
& p(\boldsymbol{\Psi}, \boldsymbol{A} \mid \boldsymbol{I}, \boldsymbol{\theta}) \propto \\
& \prod_{k=1}^{N_{f}} \prod_{n=1}^{N_{z}} \mathcal{N}\left(I_{n}\left(\lambda_{k}\right) ; \sum_{p=1}^{N_{c}} a_{(n, p)} \psi_{p}\left(\lambda_{k}\right), \sigma_{n}^{2}\right) \\
\times & \prod_{p=1}^{N_{c}} \prod_{k=1}^{N_{f}} \mathcal{G}\left(\left(\psi_{p}\left(\lambda_{k}\right) ; \alpha_{p}, \beta_{p}\right) \times \prod_{n=1}^{N_{z}} \prod_{p=1}^{N_{c}} \mathcal{G}\left(a_{(p, n)} ; \gamma_{p}, \delta_{p}\right) .\right.
\end{aligned}
$$

The estimation of the source signals and of the mixing coefficients is performed using marginal posterior mean estimator and Markov Chain Monte Carlo (MCMC) methods. These stochastic methods are extensively documented in the statistical literature (see the books [36, 37] and the references therein). All the stochastic simulation steps including the expressions of the conditional posterior distributions and their simulation techniques are detailed in [35], where this method is termed Bayesian Positive Source Separation (BPSS).

\subsection{Hyperspectral data analysis by BPSS}

A practical constraint of the Mars hyperspectral data is the high resolution of the instrument which provides a data cube of large size. Then, the computation load of the BPSS approach becomes very important and even the computation becomes almost impossible with a standard computer (need of a huge memory space, high computation time). For example, with a standard PC ( $3 \mathrm{GHz}$ and $3 \mathrm{GO}$ or RAM), it can be seen that for a data cube where the image size is $128 \times 128$ pixels, the computation time for $10^{4}$ iterations is about 4 days and 14 hours. In that respect, a reduction of the dimension is necessary before applying the BPSS approach to the hyperspectral data provided by the OMEGA instrument.

In this purpose, we suggest to process a smaller data set which is representative of the whole hyperspectral images, i.e. a selection of pixels corresponding to areas where all the chemicals existing in the whole image are present. Therefore, their pure spectra can be estimated by BPSS with a reduced computation load. Our proposal is to exploit the spatial independent component analysis results (Section 4) for selecting a few number of pixels in independent areas of the spatial coordinates, i.e. areas classified as $\mathrm{H}_{2} \mathrm{O}$ ice, $\mathrm{CO}_{2}$ ice or dust. We define the relevant pixels associated to each source as those where the contribution of this source is important. At each pixel $n$, the contribution is measured as the SNR loss, i.e. the variation of the spatial SNR when one particular source is removed from the mixture. For each chemical, we define the most relevant pixels as the first $15 \%$ with the highest spatial SNR loss and then we select randomly a fixed number (50 in the sequel for each independent component image) of pixels among this set. 


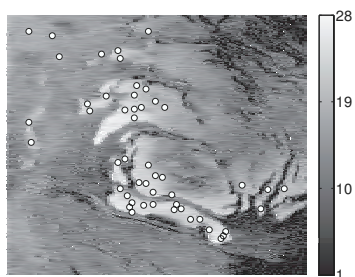

(a) Spatial SNR loss of IC4 and selected pixels

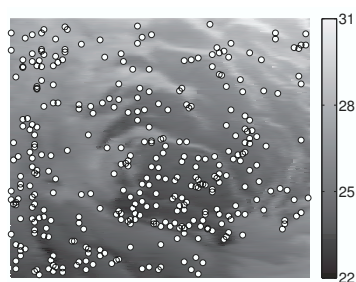

(b) Selected pixels from the whole image
Fig. 4. Illustration of the pixel selection step.

\subsection{Experimental Results}

The proposed approach is applied to the RDS hyperspectral images presented in section 2. Fig. 4 illustrates the spatial SNR loss and the selected pixels from the fourth independent component image and the selected pixels after processing of all the independent components. The results of the separation using BPSS with the mixture spectra provided by the selected pixels are post-processed to correct scale and ordering ambiguities and deduce abundance fractions. The identification of the spectra is straightforward from the correlation with the reference spectra as seen from equation (20) where the matrix entries $r_{i j}$ of the matrix $\mathbf{R}$ are the correlation coefficients between the reference spectra $\psi_{i} \in\left\{\psi_{\mathrm{H}_{2} \mathrm{O}}, \psi_{\mathrm{CO}_{2}}, \psi_{\text {dust }}\right\}$ and the estimated pure spectra $\hat{\psi}_{j} \in\left\{\hat{\psi}_{1}, \hat{\psi}_{2}, \hat{\psi}_{3}\right]$.

$$
\mathbf{R}=\left(\begin{array}{lll}
\mathbf{0 . 9 1} & 0.79 & 0.87 \\
0.72 & 0.65 & \mathbf{0 . 9 9} \\
0.89 & \mathbf{0 . 9 6} & 0.55
\end{array}\right)
$$

One can note that the correlation coefficient is very high (0.99) for the dust endmember and lower for $\mathrm{CO}_{2}$ ice and $\mathrm{H}_{2} \mathrm{O}$ ice. After scaling and permutation of the identified spectra, the reference spectra are plotted together on Fig. 5. It can be noted the similarity between the estimated spectra and the references ones. The similarity is lower for the both $\mathrm{CO}_{2}$ ice and $\mathrm{H}_{2} \mathrm{O}$ ice in the spectral region near $2 \mu \mathrm{m}$ because of the presence of a deep atmospherical band. On the contrary, the dust source is in relative better agreement with the reference spectra (see equation (5)) because both contain the atmospherical transmission.

The results show that spatial as well as spectral components extracted using positivity constraints are relevant, while the ICs extracted by ICA are spurious, as suspected since the independence assumption was wrong !

\section{CONCLUSION}

In this paper, we explained that ICA is a method which must be applied carefully for providing relevant results.

First, a physical modeling of the observation model is required for assuming the nature - linear instantaneous or con-
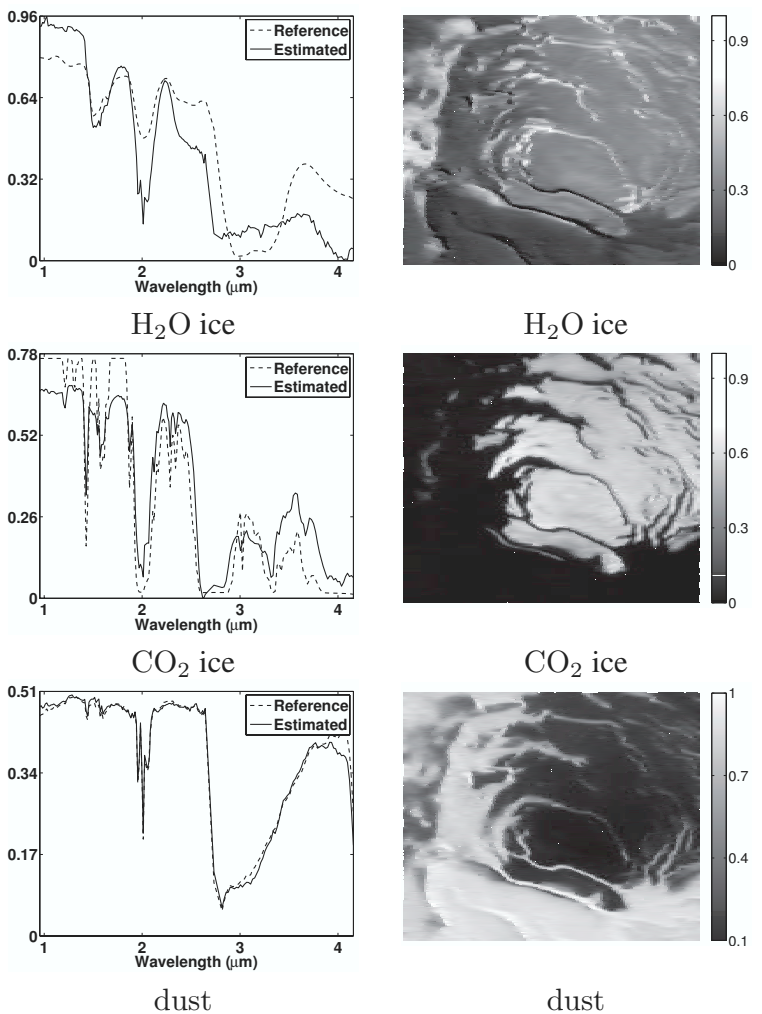

Fig. 5. Estimated and reference spectra (left) and abundance fractions (right) for the three endmembers

volutive, or nonlinear - of the mixing system and for choosing a suited separating system. Then, the determination of the IC number is often a useful step for reducing the computational load. After running an ICA algorithm, interpretation of ICs is usually not so simple. It requires priors, interactive discussions and complementary experiments with experts of the application domain.

Especially, it is important to check if ICs satisfy (i) the independence assumption, (ii) physical constraints (like spectrum positivity in this paper). For hyperspectral images, independence of spatial endmember abundances is not satisfied and it leads to wrong results: for instance, the spectrum related to one IC has negative values! For avoiding such irrelevant results, it can be much more efficient to use all the available extra information. In this paper, one successfully exploit the positivity of spectra and of mixture entries. However, locally i.e. at particular points $(x, y, \lambda)$, the approximation quality (measured as the error between the ICA approximated data and the observed data) can be poor: one may then suspect the linear model (4) to be no longer valid. At these points, further investigations assuming other endmembers (i.e. other IC) or a nonlinear model must be done. 
More generally, if possible, we recommend to consider semi-blind source separation methods. These approaches, exploiting priors even very weak like temporal correlation [38, 39], non-stationarity [40, 41], positivity or sparsity [42, 43], often leads to more efficient - sometimes simpler, too - algorithms. General frameworks exploiting priors are currently intensively explored. Methods assuming coloration and/or non-stationarity can be used in the time domain or in the frequency domain (after short term Fourier transform) and have two main advantages: (i) they lead to very efficient algorithms based on joint diagonalization of matrices, (ii) they use second-order statistics and are able to separate Gaussian sources. Although they have a high computational cost, two recent approaches are very attractive,too: (i) Bayesian source separation methods [28] for their ability to manage any prior knowledges provided than they can be stated in probabilistic terms, (ii) Sparse component analysis (SCA) [43] which can be applied when sources ${ }^{2}$ are sparse, i.e. most of the samples are close to zero, for their ability to provide solution when there are less observations than sources.

\section{REFERENCES}

[1] A. Hyvärinen, J. Karhunen, and E. Oja, Independent component analysis, Adaptive and Learning Systems for Signal Processing, Communications, and Control. John Wiley, New York, 2001.

[2] A. Cichocki and S.-I. Amari, Adaptive Blind Signal and Image Processing- Learning Algorithms and Applications, John Wiley, 2002.

[3] P. Comon, "Independent component analysis, a new concept?," Signal Processing, vol. 36, no. 3, pp. 287314, 1994.

[4] D. Yellin and E. Weinstein, "Criteria for multichannel signal separation," IEEE Trans. on Signal Processing, vol. 42, no. 8, pp. 2158-2168, Aug. 1994.

[5] H.L. Nguyen Thi and C. Jutten, "Blind separation of sources : algorithms for convolutive mixtures of large bandwidth signals," Signal Processing, vol. 45, no. 22, pp. 209-229, 1995.

[6] C. Simon, Ph. Loubaton, and C. Jutten, "Separation of a class of convolutive mixtures: a contrast function approach," Signal Processing, vol. 81, pp. 883-887, June 2001.

[7] A. Taleb and C. Jutten, "Source separation in postnonlinear mixtures," IEEE Trans. on Signal Processing, vol. 47, no. 10, pp. 2807-2820, 1999.

\footnotetext{
${ }^{2}$ or a sparsifying transform of the sources
}

[8] M. Babaie-Zadeh, C. Jutten, and K. Nayebi, "A geometric approach for separating post nonlinear mixtures," in Proc. of the XI European Signal Processing Conf. (EUSIPCO 2002), Toulouse, France, 2002, vol. II, pp. 1114.

[9] J. Eriksson and V. Koivunen, "Blind identifiability of class of nonlinear instantaneous ICA models," in Proc. of the XI European Signal Proc. Conf. (EUSIPCO 2002), Toulouse, France, Sept. 2002, vol. 2, pp. 7-10.

[10] S. Achard and C. Jutten, "Identifiability of post nonlinear mixtures," IEEE Signal Processing Letters, vol. 12, no. 5, pp. 423-426, May 2005.

[11] C. Jutten and J. Karhunen, "Advances in blind source separation (BSS) and independent component analysis (ICA) for nonlinear mixtures," International Journal of Neural Systems, vol. 14, no. 5, pp. 1-26, 2004.

[12] S. Moussaoui, H. Hauksdóttir, F. Schmidt, C. Jutten, J. Chanussot, D. Brie, S. Douté, and J. A. Benediksson, "On the decomposition of mars hyperspectral data by ICA and bayesian positive source separation," $\mathrm{Neu}$ rocomputing, 2007, in revision.

[13] J.-P Bibring et al., "Perennial water ice identified in the south polar cap of Mars," Nature, vol. 428, pp. 627-630, april 2004.

[14] F. Schmidt, S. Douté, and B. Schmitt, "Wavanglet : an efficient supervised classifier for hyperspectral images," Accepted to IEEE Trans. on Geoscience and Remote Sensing, 2007.

[15] N. Keshava and J. F. Mustard, "Spectral unmixing," IEEE Signal Processing Magazine, vol. 19, no. 1, pp. 14-57, January 2002.

[16] D. Tanre, M. Herman, P. Y. Deschamps, and A. de Leffe, "Atmospheric modeling for space measurements of ground reflectances, including bidirectional properties," Applied Optics, vol. 18, pp. 3587-3594, Nov. 1979.

[17] S. Douté, B. Schmitt, Y. Langevin, J.-P. Bibring, F. Altieri, G. Bellucci, B. Gondet, F. Poulet, and the MEX OMEGA team, "South Pole of Mars: Nature and composition of the icy terrains from Mars Express OMEGA observations," Planetary and Space Sience, vol. 55, pp. 113-133, Jan. 2007.

[18] J.-F. Cardoso and A. Souloumiac, "Blind beamforming for non Gaussian signals," IEE Proceedings- $F$, vol. 140, no. 6, pp. 362-370, 1993.

[19] D. Donoho and V. Stodden, "When does non-negative matrix factorization give a correct decomposition into 
parts?," in Advances in Neural Information Processing Systems (NIPS'2003), Cambridge, United States, 2003.

[20] S. Moussaoui, D. Brie, and J. Idier, "Non-negative source separation: Range of admissible solutions and conditions for the uniqueness of the solution," in Proc. of IEEE International Conference on Acoustics, Speech, and Signal Processing (ICASSP'2005), Philadelphia, USA, March 2005, pp. 289-292.

[21] R. Tauler, B. Kowalski, and S. Fleming, "Multivariate curve resolution applied to spectral data from multiple runs of an industrial process," Analytical Chemistry, vol. 65, pp. 2040-2047, 1993.

[22] D.D. Lee and H.S. Seung, "Learning the parts of objects by non-negative matrix factorization," Nature, vol. 401, pp. 788-791, 1999.

[23] C.L. Lawson and R.J. Hanson, Solving Least-Squares Problems, Prentice-Hall, 1974.

[24] R. Bro and S. De Jong, "A fast non-negativity constrained least squares algorithm," Journal of Chemometrics, vol. 11, pp. 393-401, 1997.

[25] P. Paatero and U. Tapper, "Positive matrix factorization: a nonnegative factor model with optimal utilization of error estimates of data values," Environmetrics, vol. 5, pp. 111-126, 1994.

[26] P. O. Hoyer, "Non-negative sparse coding," in Proc. of IEEE Workshop on Neural Networks for Signal Processing (NNSP'2002), 2002, pp. 557-565.

[27] S. A. Abdallah and M. D. Plumbley, "Polyphonic transcription by non-negative sparse coding of power spectra," in Proc. International Conference on Music Information Retrieval (ISMIR'2004), Barcelona, Spain, Oct 2004, pp. 318-325.

[28] A. Mohammad-Djafari, "A Bayesian approach to source separation,” in MaxEnt, J. T. Rychert, G. J. Erickson, and C. R. Smith, Eds., Boise, Idao, 1999, vol. 567, pp. 221-244, AIP Conference Proceedings.

[29] D.B. Rowe, Multivariate Bayesian Statistics: Models for Source Separation and Signal Unmixing, CRC Press, Boca Raton, Florida, USA, 2003.

[30] H. Snoussi and J. Idier, "Bayesian blind separation of generalized hyperbolic processes in noisy and underdeterminate mixtures processes in noisy and underdeterminate mixtures," IEEE Trans. Signal Processing, vol. 54, no. 9, pp. 3257-3269, September 2006.

[31] M. F. Ochs, R. S. Stoyanova, F. Arias-Mendoza, and T. R. Brown, "A new method for spectral decomposition using a bilinear Bayesian approach," Journal of Magnetic Resonance, vol. 137, pp. 161-176, 1999.
[32] J. Miskin and D. MacKay, "Ensemble learning for blind source separation," in S. Roberts and R. Everson, editors, Independent Component Analysis: Principles and Practice. 2001, pp. 209-233, Cambridge University Press.

[33] S.J. Roberts and R. Choudrey, "Data decomposition using independent component analysis with prior constraints," Pattern Recognition, vol. 36, pp. 1813-1825, 2003.

[34] S. Moussaoui, D. Brie, C. Carteret, and A. Mohammad Djafari, "Application of Bayesian non-negative source separation to mixture analysis in spectroscopy," in MaxEnt, R. Fischer, R. Preuss, and U. von Toussaint, Eds., Garching, Germany, July 2004, vol. 735, pp. 237-244, AIP Conference Proceedings.

[35] S. Moussaoui, D. Brie, A. Mohammad-Djafari, and C. Carteret, "Separation of non-negative mixture of nonnegative sources using a Bayesian approach and MCMC sampling," IEEE Trans. Signal Processing, vol. 54, no. 11, pp. 4133-4145, November 2006.

[36] C.P. Robert, Monte Carlo Statistical Methods, SpringerVerlag, 1999.

[37] M.-H Chen, Qi-Man Shao, and J. G. Ibrahim, Monte Carlo Methods in Bayesian Computation, SpringerVerlag, New York, 2000.

[38] L. Tong, V. C. Soon, Y.-F. Huang, and R. Liu, "Amuse: A new blind identification algorithm," in Proc. IEEE ISCAS, New Orleans, LA, May 1990, pp. 1784-1787.

[39] A. Belouchrani, K. Abed-Meraim, J.-F. Cardoso, and E. Moulines, "A blind source separation technique using second-order statistics," IEEE, Trans. on Signal Processing, vol. 45, no. 2, pp. 434-444, Feb. 1997.

[40] K. Matsuoka, M. Ohya, and M. Kawamoto, "A neural net for blind separation of nonstationary signals," Neural Networks, vol. 8, no. 3, pp. 411-419, 1995.

[41] D. T. Pham and J.-F. Cardoso, "Blind separation of instantaneous mixtures of nonstationary sources," IEEE Trans. on Signal Processing, vol. 49, no. 9, pp. 18371848, 2001.

[42] M. Zibulevsky and B.A. Pearlmutter, "Blind source separation by sparse decomposition in a signal dictionary," Neural Computation, vol. 13, no. 4, pp. 863-882, 2001.

[43] R. Gribonval and S. Lesage, "A survey of sparse components analysis for blind source separation: principles, perspectives and new challenges," in Proc. ESANN 2006, Bruges, Belgium, April 2006, pp. 323-330. 